\title{
EDICIÓN ACADÉMICA EN LA ERA DIGITAL: MODELOS, DIFUSIÓN Y PROCESO DE INVESTIGACIÓN
}

\author{
Paul Spence (King's College London)
}

Fecha de recepción: 14-10-2013 / Fecha de aceptación: 01-12-2013

Cita REComendada: Paul Spence, «Edición académica en la era digital: modelos, difusión y proceso de investigación, Anuario Lope de Vega. Texto, literatura, cultura, XX (2014), pp. 47-83.

DOI: <http://dx.doi.org/10.5565/rev/anuariolopedevega.74>

\section{RESUMEN}

Este artículo analiza los retos para la edición académica en la era digital, tomando una perspectica analítica y práctica, con referencia a la historia reciente en la edición digital, y sobre todo a experiencias dentro de investigadores en las humanidades digitales.

Palabras clave: Humanidades Digitales; edición digital; cuestiones textuales.

\section{Abstract}

This article analyses scholarly edition in the digital age from both theoretical and practical perspectives, with reference to recent history in digital edition, and with special reference to research in this area in the field of digital humanities.

KeYworDs: Digital humanities; digital edition; text-based scholarship. 
Oupongo que no es controvertido afirmar a estas alturas que estamos viviendo cambios radicales en la forma de producir, recibir y compartir la cultura a causa de la era digital. La tecnología ha revolucionado la publicación de los textos, es decir, el proceso que comienza cuando tradicionalmente un investigador manda su texto a una casa editorial para ser sometido al proceso de producción y difusión de las obras científicas, pero no se han visto, por ahora, reajustes semejantes en el mundo académico de las humanidades, y mucho menos en el proceso de editar (o «reconstruir») un texto donde nuestros métodos para buscar las pruebas materiales, contrastarlas, registrar nuestras dudas e interpretaciones, y después plasmarlas en un texto presentado al mundo exterior, no han variado mucho en términos globales, fuera del uso generalizado del correo electrónico, internet, y hasta cierto punto, los medios sociales. Estas herramientas han supuesto una transformación parcial en la "comunicación erudita» ${ }^{1}$ en las ciencias humanas, pero es de un grado muy inferior a las transformaciones que se han visto en otras áreas de la investigación, como las ciencias empíricas o naturales, donde la era digital ha provocado un replanteamiento de base en el proceso de la investigación en varias facetas. ${ }^{2}$

\section{UNA PERSPECTIVA ACADÉMICA SOBRE LOS MODELOS Y MARCOS DIGITALES PARA LA EDICIÓN}

Aunque es evidente que muchos investigadores se dieron cuenta muy temprano del potencial de los instrumentos digitales para mejorar el proceso de preparación de una edición especializada (P. D. A. Harvey 2001, Sánchez Prieto Borja 1998), y hay una rica historia de investigación experimental en edición digital (Schreibmann 2013), sobre todo asociada al campo de las «Humanidades Digitales», esta inquietud no se ha concretado en pasos prácticos, salvo en algunos focos donde los recursos o un interés especial lo han alentado. Hace casi diez años, Peter Robinson [2005] opinaba que todavía muchos investigadores seguían sin estar convencidos de que los avances en la

1 Utilizo aquí "comunicación erudita» como traducción del término inglés "scholarly communication», que describe el proceso por el que los investigadores académicos crean, comparten, diseminan y preservan sus conocimientos relacionados con la enseñanza y la investigación, bien sea en la producción de publicaciones formales o en otros aspectos de la vida académica.

2 Véanse, por ejemplo los cambios en la difusión ("What the journal did for a single, formal product (the article), the Web is doing for the entire breadth of scholarly output», afirma Jason Priem 2013) o en el tratamiento de los materiales de la investigación (Borgman 2009, «Many of the sciences, especially those "big science" areas that require large scale instrumentation and produce vast volumes of data, are in transition to a data-driven paradigm»). 
tecnología digital ofrecían una ruta asequible para la «edición académica», ${ }^{3}$ y si bien ha habido modificaciones importantes en algunas de las condiciones subyacentes en la edición digital — cambios, por ejemplo, en la lectura en pantalla, en la percepción de recursos digitales y en los modelos económicos de la edición en general- muchas dudas históricas siguen sin resolverse. Procesos de gran transformación, como los que estamos viviendo, pueden provocar entusiasmo y expectación, pero también incertidumbre y ansiedad. Lejos de enfrentarse a, o incluso encauzar, estos procesos, los editores e investigadores en humanidades generalmente han preferido una actitud pasiva y defensiva. Las ediciones impresas - hoy en día producidas con herramientas digitales, aunque sean procesadores de texto como Microsoft Word — siguen las pautas del modelo impreso con poca variación, y más sorprendentemente, la mayoría de las ediciones digitales producidas por ahora, incluso las provenientes de las humanidades digitales, muestran fuertes vinculaciones con la "plantilla impresa», pese a su vocación orientada a conseguir mayor fluidez y conectividad digital.

Yo diría que estamos viviendo un momento histórico distinto al descrito por Robinson y hay una conciencia general ahora, a diferencia de hace diez años, de que «hay que hacer algo», y además con cierta urgencia — pues como argumentan Baraibar y Cohen, «hay que estar presente en los medios y soportes en los que, en pleno siglo XXI, se está dando la comunicación y la conversación académicas y sociales» (Baraibar 2012:159)—. Bien, somos conscientes de la necesidad de un cambio de dirección, pero ¿hacia qué destino? ¿Y cómo llegamos a él? En cierta medida, la desidia hacia las innovaciones digitales se puede explicar por el hecho de que la persona encargada de una edición académica que se quiera lanzar al campo de lo digital encuentra terreno resbaladizo, accidentado, que implica tantos riesgos como oportunidades. En lugar de ofrecer una única alternativa estable, el mundo digital ofrece múltiples opciones que sufren modificaciones continuas.

Conviene definir algunos límites sobre este análisis de la «edición digital», un término que engloba varios conceptos distintos, desde la mera publicación, en formato digital, de una copia facsimilar de un periódico ( $u$ otro objeto cultural), normalmente accesible en red, hasta ediciones especializadas usando todas las capacidades - de representación, de visualización y de divulgación — de la tecnología digital. Esta misma

3 Es difícil encontrar un término adecuado para el tipo de edición digital que se propone en este artículo, que más o menos se aferra al concepto de «scholarly digital edition» en inglés. He empleado «edición académica» o a veces "edición digital académica» para evitar la carga teórica y práctica de «edición crítica» y otros términos utilizados. 
variación en el uso del término «edición digital» y las amplias posibilidades de funcionalidad que existen incluso en el ámbito especializado dificultan el debate, por lo que ahora paso a resumir, con una perspectiva humanística, algunas de las características principales de los modelos y marcos digitales a nuestro alcance en estos momentos.

\subsection{TeXto Digital enRiquecido: TEI/XML}

Nuestro estudio empieza por la marcación digital. Los sistemas de puntuación o diseño de un documento representan formas de marcar un texto para su mejor comprensión, y cualquier editor estará acostumbrado a trabajar con marcas, que pueden tener valores semánticos o de presentación, y que pueden servir para etapas de validación de un texto (corrección de pruebas) o incluso en el proceso de preparación / publicación en su conjunto, como un aparato crítico tradicional. ${ }^{4}$ De esta manera, el editor académico (por ejemplo, de la tradición ecdótica) está acostumbrado a trabajar con una serie de fórmulas y convenciones que resultan desconcertantes para un lector medio, pero que son imprescindibles para un investigador que quiera conocer en detalle la historia y las variaciones de un texto.

Los últimos veinte años de investigación en la representación del texto en formato digital han producido varios paradigmas, pero actualmente muchos editores con formación en las humanidades digitales favorecen lo que voy a llamar «modelo TEI/XML». ${ }^{5}$ A nivel técnico y formal, este modelo está compuesto por un estándar internacional para intercambiar datos (XML) y un marco internacional para representar textos en las ciencias humanas de manera digital (TEI), pero en realidad encarna una compleja confluencia de agentes, teorías, metodologías, herramientas y prácticas para representar el texto en formato digital. Si bien no tenemos espacio para entrar aquí en detalle sobre la naturaleza de estas tecnologías o de sus ventajas / desventajas, ${ }^{6}$ sí conviene resumir algunos puntos clave.

En lugar de pretender reconstruir simplemente la presentación original de un texto (esto se puede hacer posteriormente en otro proceso), imitando las convenciones tipográficas o de diseño de página del original, el modelo TEI/XML ofrece

4 Véanse, por ejemplo, los signos convencionales usados en los criterios de edición del grupo PRolope para correcciones manuscritas en un aparato crítico: $</>$, para adición en la línea; $<\backslash>$, para adición en la entrelínea, etc. (Prolope 2008:57 o MacKenzie 1997).

5 «TEI is the lingua franca of digital scholarly editing on a global basis», asevera Mueller [2011].

6 Compárense Renear [2004] y Fiormonte [2010], por ejemplo. 
una representación estructurada, sustituyendo formato de presentación (cursiva, cabecera de mayor tamaño, etc.) por unas marcas semánticas que captan los distintos elementos significantes (para el editor) a un nivel conceptual. Esta separación entre una representación teórica del texto y su presentación tiene varias ventajas prácticas, entre ellas la posibilidad de poder publicar desde un mismo texto «fuente» en varios formatos (PDF, HTML, ePub) usados para las publicaciones impresas y digitales; poder «exponer» los materiales a otros procesos / programas informáticos; la posibilidad de ofrecer múltiples versiones del mismo texto en un mismo formato (por ejemplo, edición crítica y edición diplomática); facilitar materiales auxiliares, como índices onomásticos o apéndices (listas de los personajes que hablan por acto en una obra teatral, por ejemplo), y para terminar, la preservación.

Una de las mejores pruebas de su versatilidad como formato para representar textos humanísticos es el uso de un elemento TEI con marcas < choice $>$ y $<$ / choice $>$ para demostrar alternativas dentro del mismo texto. Para dar un ejemplo, si queremos representar la normalización de abreviaturas manifestada en el paso de «quãdo» a «cuando» (Prolope 2008:31), podemos usar la siguiente marcación, que capta tanto el texto original como su desarrollo llevado a cabo por el editor, posibilitando múltiples usos posteriores, a la vez que deja constancia verificable de la labor filológica.

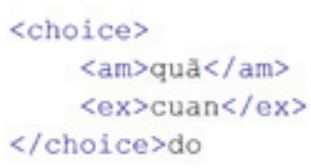

Figura 1. Ejemplo de abreviatura en XML, tomado de los criterios de edición del grupo Prolope (2008); <am> demuestra el texto reemplazado y <em> el texto desarrollado/sustituido.

De esta manera, se ofrece la posibilidad de mantener todas las variantes e interpretaciones en un solo texto, lo cual puede resultar más eficaz, aunque también más cuestionable si la densidad de las interpretaciones es muy alta en un determinado fragmento. Esto ejemplifica uno de los retos principales de la edición digital: cómo y dónde señalar la interpretación de un texto. Un caso interesante es el aparato crítico, cuya funcionalidad se puede sustituir o reconstruir de varias maneras en TEI, y donde un grupo de especialistas actualmente contempla varios niveles: (1) representar las unidades del cotejo a través de los testimonios; (2) marcar 
los enlaces y el alineamiento entre los fragmentos de las variantes; y (3) marcar la representación para derivar el aparato del cotejo. ${ }^{7}$

Esto nos hace replantear cuestiones filológicas básicas: ¿Se pueden (o se deben) representar todas la anotaciones filológicas en un solo texto? ¿Es aconsejable buscar una manera de separar los distintos niveles (o distintas capas) de marcación / interpretación? ¿Se pueden generalizar las tareas de la edición especializada hasta cierto punto o deben ser vistas como tareas cuyas características cambian según las necesidades de cada proyecto de investigación? ¿Y hasta qué punto se puede separar la investigación misma de una salida (publicación) concreta?

TEI ofrece modelos de marcación sofisticados y bien documentados para la gran mayoría de los casos imaginables en las ciencias humanas, y cuando no hay solución, existen mecanismos probados para sugerir nuevos casos, o incluso para la personalización. ${ }^{8}$ Este modelo ha sido usado en varios proyectos - tan variados como la edición digital de La entretenida, ${ }^{9}$ Perseus Digital Library, ${ }^{10}$ Menota (Medieval Nordic Text Archive), ${ }^{11}$ The Orlando Project, ${ }^{12}$ Henrik Ibsens Skrifter ${ }^{13}$ y Early Americas Digital Archive ${ }^{14}$ — pero sigue habiendo barreras importantes para los recién llegados, o para los que no tienen acceso a apoyo técnico para la transformación de una edición TEI en uno de los formatos comunes de publicación, aunque iniciativas como TEI By Example, ${ }^{15}$ TAPAS Project (TEI Archiving Publishing and Access Service) ${ }^{16}$ y TEI Boiler Plate ${ }^{17}$ han empezado a cerrar, en parte, esta brecha.

Un porcentaje alto de las opiniones y los comentarios sobre TEI se centra en su uso como modelo de representación primario (es decir, el formato usado en el mismo momento de preparar una edición) y en el caso en el que alguien

7 http://wiki.tei-c.org/index.php/Critical Apparatus Workgroup.

8 Se puede personalizar por proyecto, como es el caso de Gascon Rolls (1317-1468) (http://gasconrolls.org), o por comunidades científicas, como EPIDOC (http://sourceforge.net/p/epidoc/wiki/ Home/) para la epigrafía, Best Practices for TEI in Libraries (http://tei-c.org/SIG/Libraries/teiinlibraries/) para biblioteca, etc.

9 http://entretenida.outofthewings.org/.

10 http://www.perseus.tufts.edu/.

11 http://www.menota.org/forside.xhtml.

12 http://www.artsrn.ualberta.ca/orlando/.

13 http://www.ibsen.uio.no/forside.xhtml.

14 http://mith.umd.edu/eada/intro.php.

15 http://www.teibyexample.org/.

16 http://tapasproject.org/.

17 http://dcl.slis.indiana.edu/teibp/. 
edita un texto viendo todas sus marcas sin instrumentos de ayuda (esto ignora el hecho de que se pueden crear interfaces que efectivamente reproducen un entorno de edición «tradicional»). Pero la realidad es mucho más compleja —algunos editan en Word o en una base de datos y luego exportan sus materiales a TEI/XML, o editan en entornos digitales que automáticamente crean versiones TEI de sus materiales (Muñoz Pons 2013)—. El debate sobre TEI/XML pierde sentido si no se establece en qué fases del proceso de edición lo estamos utilizando — preparación, revisión y corrección, producción de una publicación, preservación, etc.-

\section{div head 1.2. hi rend="bold" Display of Verse hi head}

p In order to make the verse form clearer, the practice of abbreviating character names and embedding them within the text, which may be observed in the first edition of 1615 , has been followed, but with the names rendered in bold for ease of viewing. Shared lines are, accordingly, not split, as is usually the case in modern printed editions. Stanzas are separated by a space, again with a view to highlighting the verse form. $\mathrm{p}$ div

Figura 2. Ejemplo de texto en un editor XML, ocultando marcas (de la edición digital La entretenida).

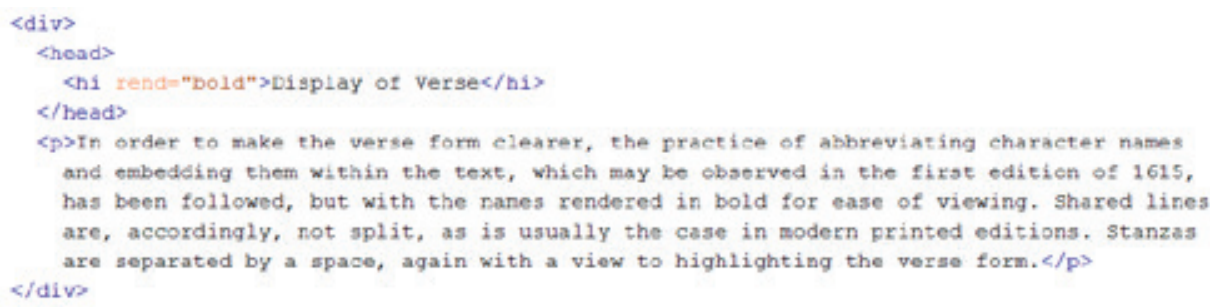

Figura 3. Ejemplo de texto en un editor XML, con marcas (de la edición digital $L a$ entretenida).

Parece contradictorio que, aunque XML ha tenido más éxito en procesos de producción (hacia un "producto» final, como en el mundo de la publicación digital) ${ }^{18}$ que en la investigación, su influencia teórica y práctica en el área de

18 Para una evaluación de sus éxitos y limitaciones, véase http://thefutureofpublishing. com/2012/10/xml-failed-publishing/. 
la edición digital de textos ha afectado principalmente a su capacidad de representación primaria y almacenamiento en archivos virtuales, en lugar de su presentación final en una interfaz accesible para los usuarios, tal y como señalan Fraistat y Jones. ${ }^{19}$ Aunque el modelo TEI/XML ofrece una ruta viable para representar procesos complejos de edición, se necesita más investigación sobre las distintas fases en el proceso editorial y sobre la relación entre el modelo técnico de un texto y su realización en interfaces de preparación y presentación final de una edición.

\subsection{Marcos digitales CReados para Proyectos concretos}

Si bien algunos han apostado por una inmersión profunda en la marcación descriptiva, otros han preferido crear sistemas personalizados para comunidades o incluso para proyectos concretos. Este enfoque no necesariamente implica un rechazo frontal al uso de estándares como TEI —como hemos visto, se pueden crear sistemas que empleen TEI como modelo de base, o que al menos exporten TEI para efectos de preservación-, pero ponen el acento en el diseño de una interfaz más «amigable», que esconda la «maquinaria» tecnológica (por ejemplo, las marcas TEI) del editor, ${ }^{20} \mathrm{o}$ al menos minimice el compromiso de formación necesaria. Este es el caso del proyecto Early English Laws financiado por el AHRC, que a partir de la investigación que un equipo de $\mathrm{DDH}^{21}$ en King's College London hizo para el proyecto Out of the Wings, ${ }^{22}$ ha creado un marco integrado para la edición de varios textos legales representativos de la época medieval inglesa, ${ }^{23}$ presentando facsímiles, textos editados, aparato crítico, transcripciones, traducciones y referencias secundarias en una edición digital que permite hacer búsquedas, filtrar resultados, contraponer ventanas distintas

19 «The field of electronic textual editing currently is far more advanced in theorizing and producing the "back end" of editions - where the issues primarily involve rigorous structural markupthan it is in attending to issues relating to the "front end"» (Fraistat y Jones 2009:10).

20 Vanhoutte y otros cuestionan ese rechazo de algunos a enfrentarse a las marcas de XML, afirmando que las marcas XML son herramientas humanísticas más que técnicas y parte importante del proceso de edición (Vanhoutte 2011).

21 Department of Digital Humanities (Departamento de Humanidades Digitales de King's College London), http://www.kcl.ac.uk/ddh

22 Explicado en Spence [2013].

23 "Early English Laws is a project to publish online and in print new editions and translations of all English legal codes, edicts, and treatises produced up to the time of Magna Carta 1215» (http:// www.earlyenglishlaws.ac.uk/). 
y visualizar las relaciones entre leyes con una trasmisión hereditaria compleja. Esto permite un análisis cercano de los textos, y a la vez una visión más global, para buscar similitudes y diferencias entre versiones distintas, y facilita una navegación fluida por los materiales de investigación, donde uno puede cambiar el enfoque según sus intereses.

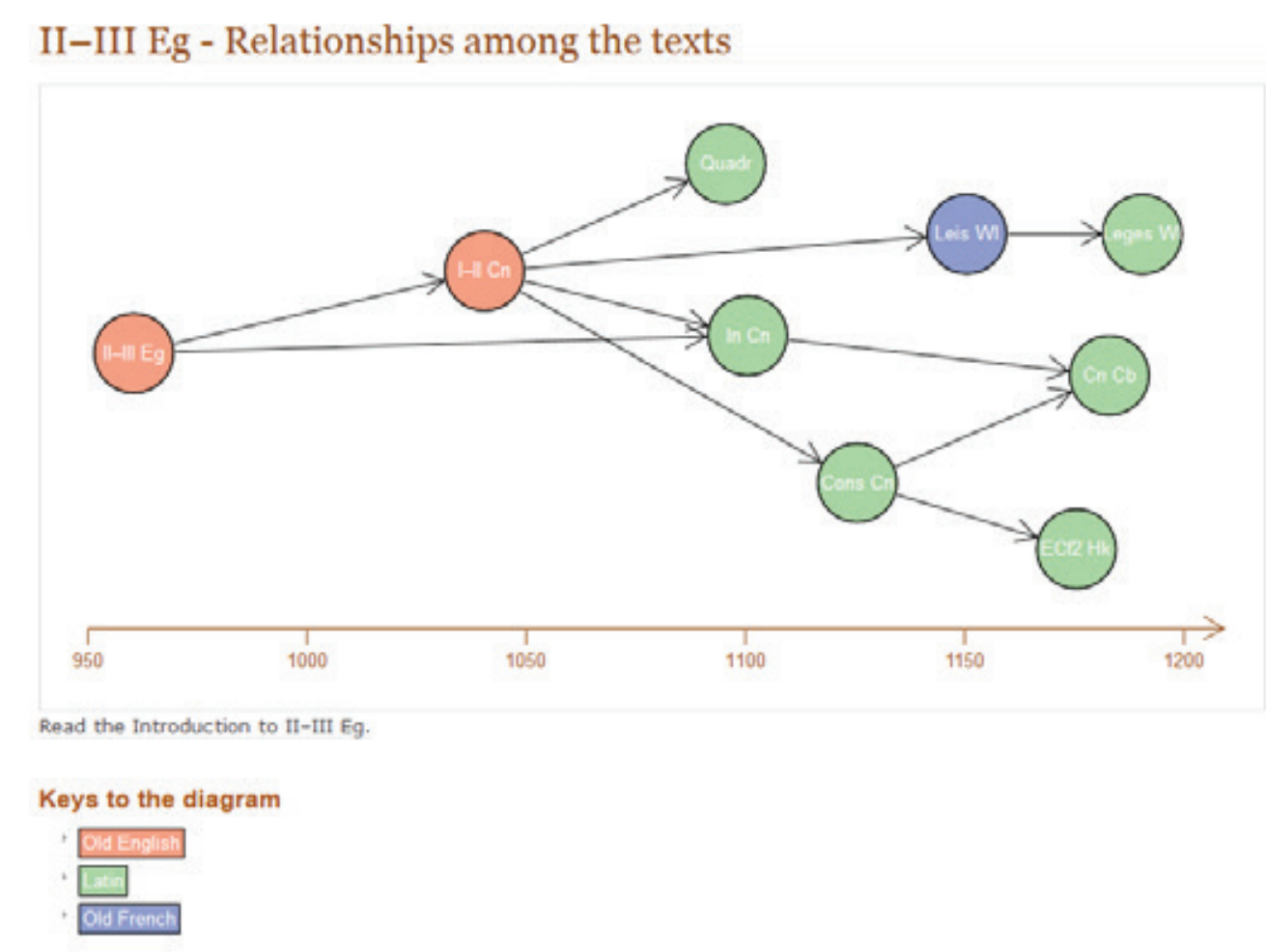

Figura 4. Visualización de la relación entre textos en el proyecto Early English Laws http://www.earlyenglishlaws.ac.uk/laws/texts/iiiii-eg/relationships/

El proyecto creó una interfaz que permite contraponer, a través de dos ventanas, los componentes distintos de la edición (por ejemplo comparar una edición con un facsímil del texto original, o, la opción por defecto, ver la edición a la izquierda y los comentarios editoriales a la derecha) y desvelar / ocultar algunas perspectivas de la investigación (aparato crítico, comentarios del editor, comentarios de otros usuarios y la organización numérica de los textos en capítulos). 


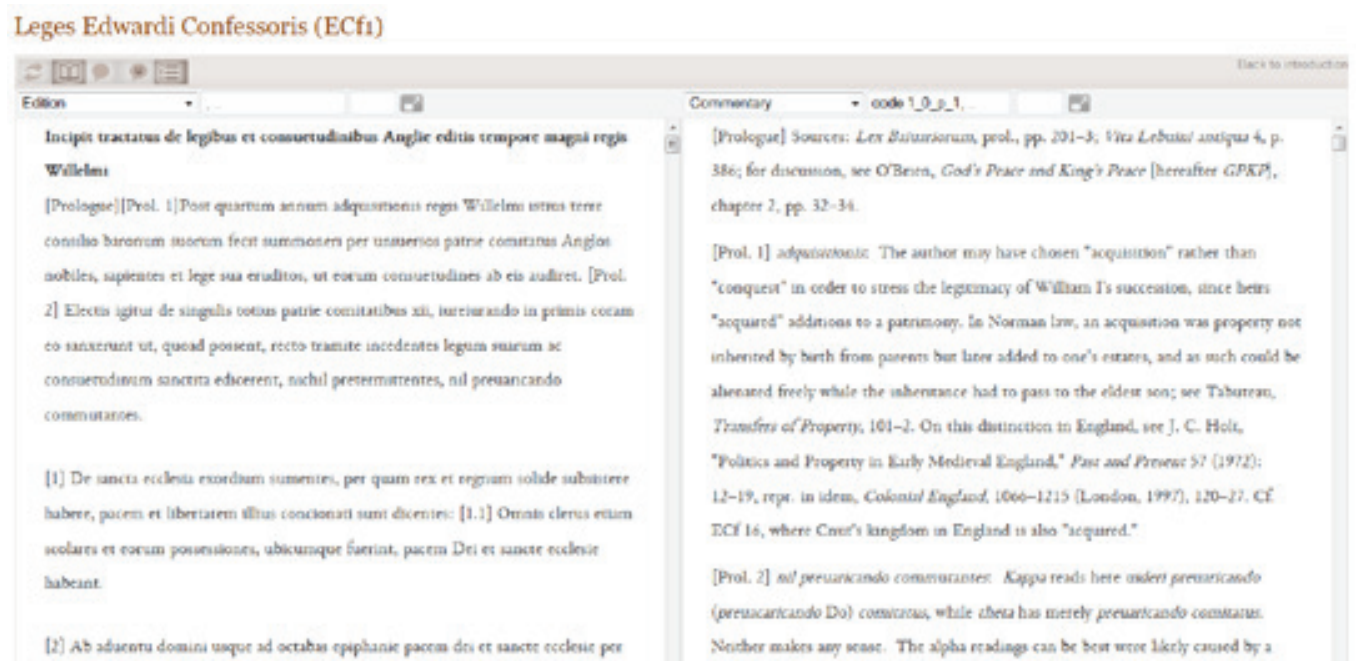

Figura 5. Interfaz en el proyecto Early English Laws que permite contraponer ventanas con edición y comentarios http://www.earlyenglishlaws.ac.uk/laws/texts/ecf1/ view/\# edition, $1 /$ commentary, 10 p 1

Early English Laws responde en buena medida a las críticas de que las ediciones digitales se limitan a seguir el patrón de la tradición impresa (y además trascienden las limitaciones de esta última), pero quizás lo más significativo, para el debate actual, es el sistema que se creó para la preparación de la edición. A diferencia de otros proyectos realizados por DDH, el ya citado Gascon Rolls (1317-1468), donde se ha formado a un equipo especializado en editar con TEI con bastante éxito, Early English Laws quiso contar con editores y equipos editoriales con una amplia distribución geográfica (además, se solicitaron propuestas de edición en un proceso público y abierto), dificultando así una formación exhaustiva o un apoyo técnico muy cercano. Se quiso aprovechar las innovaciones de la edición digital más especializada a través de un sistema accesible al editor con una formación técnica media, pudiendo gestionar el proceso de la edición a través de varias fases, donde se dejara rastro de las decisiones tomadas y el estado de cada texto. La edición se hace en línea, lo que quiere decir que se ven los cambios en tiempo real y se deja a los editores generales del proyecto acceder a los distintos estados de cada edición, antes de "publicarlos». La interfaz del editor reproduce los gestos y metáforas de un procesador de textos, con la estructura del texto en una columna a la izquierda, los comentarios en la parte inferior de la pantalla, y con botones de pantalla en la sección principal para anotar la estructura (código legal, capítulo, prólogo, sección, salto de página, aparato crítico) e interpretación editorial (título, rúbrica, texto añadido, texto borrado, espacio, 
texto añadido por el editor). Este enfoque minimiza (o quizás solamente posterga) una formación nueva de carácter «tecno-humanístico», permitiendo al editor, en gran medida, aplicar códigos entendibles por un ordenador sin tener que enfrentarse a los detalles técnicos; ahora bien, esto pierde eficacia cuanto más compleja es la estructura de la edición, sobre todo si tiene fragmentos de texto con estructura "profunda», con un gran anidamiento o solapamiento de conceptos y anotaciones.

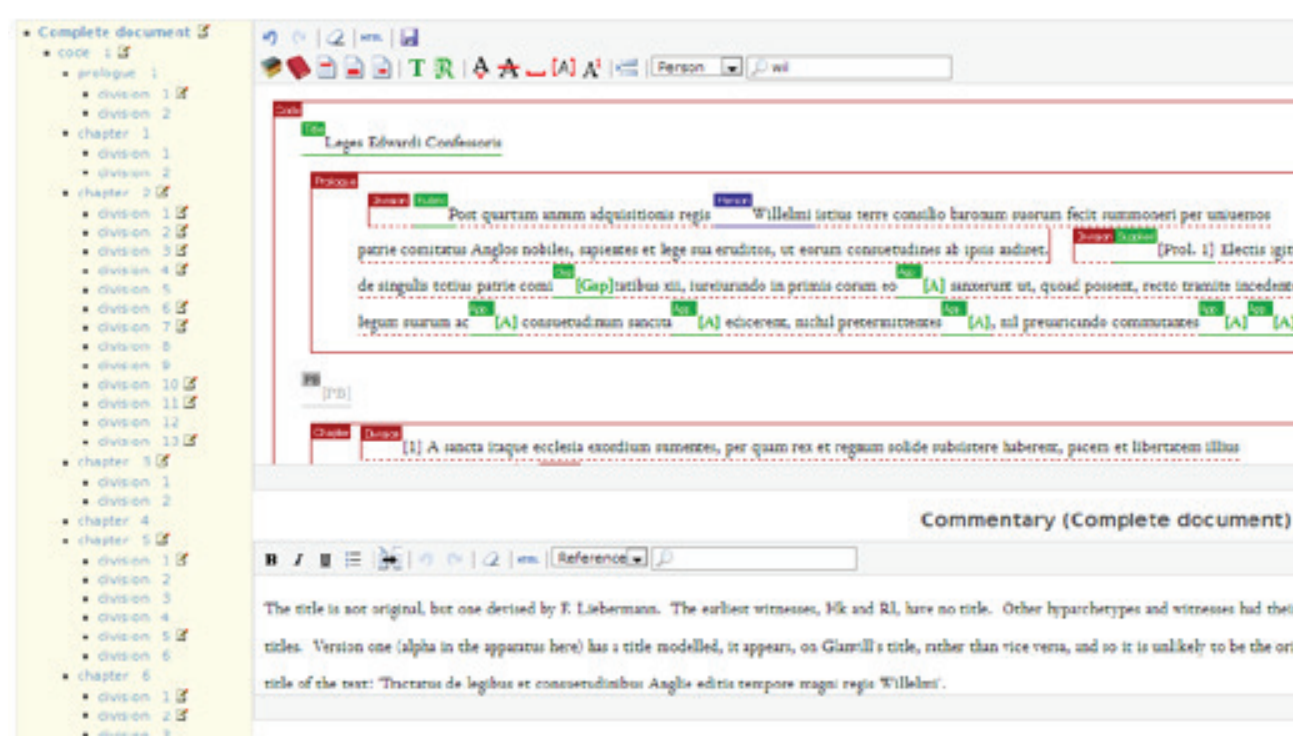

Figura 6. Interfaz de editor, proyecto Early English Laws.

Las desventajas de crear un marco para un proyecto concreto son varias: la personalización es cara en términos de recursos, que rara vez permiten mantenerse fuera de su periodo de financiación (es decir, no son sostenibles) e implican mucha repetición y solapamiento de esfuerzos entre proyectos. Pero un aspecto importante de un proyecto como Early English Laws es que pretende integrar todos los materiales de investigación necesarios para una visión global, y más allá de las versiones de texto y su interrelación, es notable el hecho de que desarrolla listas de registros de autoridad para varios aspectos de la investigación, como los códigos usados para las leyes estudiadas, sus categorías o los reinos donde aparecen. Esto constituye un compromiso con las herramientas digitales que se extiende más allá de la mera transcripción de un texto. Aunque no puede considerarse un «entorno virtual de investigación» ${ }^{24}$ en el sentido formal, comparte muchas de sus caracterís-

24 Del inglés «virtual research environment», donde los informes del programa de Jisc señalan que una definición del término es problemática (http://www.jisc.ac.uk/whatwedo/programmes/vre.aspx). 
ticas, y un paso lógico (por las razones económicas antes expuestas) sería la creación de marcos de este tipo para determinadas comunidades académicas, una vía que examinaremos con detalle más adelante.

\subsection{HerRamientas GENÉRICAS}

Una posible respuesta al argumento económico es crear o usar herramientas genéricas, y de hecho ya ha habido varios intentos de ofrecer herramientas genéricas para la edición digital especializada. Algunos, como Classical Text Editor (Hagel 2007:84), siguen la tradición de la edición crítica en buena medida; si bien CTE, en teoría, puede publicar un texto en papel y en digital (y ofrece exportación a TEI y otros formatos), históricamente ha sido utilizado más como instrumento para crear ediciones impresas que ediciones digitales (Hagel 2007:78); mientras que otros, como TUSTEP, ${ }^{25}$ siguen una conceptualización más genérica del tratamiento de texto desde una perspectiva erudita (desde la captación de datos hasta su publicación en formato impreso o digital) ${ }^{26}$ y bajo una metodología modularizada y exportable. ${ }^{27}$

Otras herramientas tienen un enfoque más específico: anotar imágenes —el UVic Image Markup Tool ${ }^{28}$ o TILE $^{29}$-, editar en un contexto social / participativo (siguiendo una metodología crowd-sourcing) ${ }^{30}$ - Scripto $^{31}$ o el marco creado para el proyecto Transcribe Bentham ${ }^{32}$ - o publicar y archivar textos editados en XML, como Kiln. ${ }^{33}$ La mayoría de las herramientas creadas hasta ahora funcionan desde el escritorio, aunque actualmente existe cierta tendencia a investigar en entornos en línea -donde herramientas como CWRC Writer ${ }^{34}$ rozan con los límites técnicos

25 http://www.tustep.uni-tuebingen.de/tustep eng.html.

26 "It contains modules for all stages of scholarly text data processing, starting from data capture and including information retrieval, text collation, text analysis, sorting and ordering, rule-based text manipulation, and output in electronic or conventional form (including typesetting in professional quality)» (http://www.tustep.uni-tuebingen.de/tustep eng.html).

27 http://www.tustep.uni-tuebingen.de/tustep ox92.html.

28 http://tapor.uvic.ca/ mholmes/image markup/.

29 http://mith.umd.edu/tile/.

30 http://iber.library.uu.nl/index.php/lq/article/view/URN\%3ANBN\%3ANL\%3AUI\%3A10-1-113603.

31 http://scripto.org/.

32 http://blogs.ucl.ac.uk/transcribe-bentham/.

33 http://github.com/kcl-ddh/kiln.

34 http://www.dh2012.uni-hamburg.de/conference/programme/abstracts/cwrc-writer-an-inbrowser-xml-editor/. 
del navegador de internet-y otras, como CATMA ${ }^{35}$ y Juxta, ${ }^{36}$ ofrecen versiones en los dos entornos.

Es evidente que la gran mayoría de estas herramientas no tienen mucha acogida y no pasan de ser más que experimentos interesantes, en buena parte por la falta de compromiso del humanista medio con la innovación tecnológica y la falta de investigación centrada en el usuario (humanista) por parte de los autores de las herramientas creadas (Gibbs y Owens 2012). Si bien es cierto que las herramientas genéricas a menudo se ajustan mal a las necesidades académicas, también observamos que las herramientas sufren problemas de presentación, sostenibilidad, interoperabilidad, apoyo y formación técnica.

\subsection{Macroinfraestructura/eScience}

Herramientas como CTE y TUSTEP favorecen la idea de editar en un solo entorno, lo cual tiene la ventaja de ofrecer cierta coherencia interna, pero otras voces opinan que la solución es crear entornos más flexibles, donde se puedan compartir e integrar tanto los materiales como las herramientas de investigación en ciencias humanas, aunando recursos e infraestructura para evitar la redundancia. Es el espíritu de la «llamada a la acción» de Christine Borgman de 2009, quien imploró a los investigadores en humanidades que crearan la misma infraestructura técnica, social y política de la que disponen las ciencias naturales y físicas bajo el modelo de la «ciberinfraestructura» o «eScience», que afecta tanto a los procesos de investigación y publicación como al concepto de materiales o «datos» de estudio (Borgman 2009). Más recientemente, un informe de la European Science Foundation ${ }^{37}$ hizo varias recomendaciones para crear una infraestructura física y digital para la investigación en humanidades que contemplaba una visión más global y estratégica, fomentando consorcios y redes interdisciplinares e internacionales (como DARIAH ${ }^{38}$ y $\mathrm{CLARIN}^{39}$ ), y promocionando el reconocimiento académico de un nuevo modelo de investigación en las ciencias humanas que acepte el carácter procesal de las publicaciones digitales y la naturaleza cada vez más colaborativa de la investigación en la sociedad de la información.

35 http://www.catma.de/.

36 http://www.juxtasoftware.org/.

37 http://www.esf.org/fileadmin/Public documents/Publications/spb42 RI DigitalHumanities.pdf.

38 http://dariah.eu/.

39 http://www.clarin.eu/. 
El concepto de infraestructura a gran escala nos conduce a entornos virtuales que integran investigadores, contenidos y tecnología, y que supondrían cambios de fondo en la manera de investigar y difundir los resultados de la investigación. Este modelo está ejemplificado en entornos virtuales para la investigación como TextGrid, ${ }^{40}$ que pretende crear una plataforma para unir en un solo repositorio los materiales de investigadores en humanidades (que pueden gestionar el grado de acceso a otros investigadores) y localizar las herramientas digitales, de manera que un investigador pueda combinar herramientas en un entorno unificado e interactivo sin mayor necesidad de formación especializada. El concepto de la ciberinfraestructura no ha cuajado tan rápidamente en las humanidades, por razones políticas (las instituciones académicas nacionales no le han prestado la atención dada a las ciencias físicas y naturales), conceptuales (el enfoque de las humanidades hacia la «interpretación») y académicas (no se le ha dado el mismo apoyo desde las mismas instituciones de humanidades), y hay serias dudas sobre su sostenibilidad económica (Hedges et al. 2013:8), pero en caso de ganar terreno, promete tener un efecto importante sobre el aspecto práctico de la investigación en las humanidades.

\subsection{HeRRAMIENTAS CREADAS POR Y PARA LAS «COMUNIDADES DE PRÁCTICA»}

Por ahora el modelo más exitoso en las humanidades, en muchos casos, sigue siendo (donde resulta factible) la «comunidad de práctica», una comunidad autodefinida en términos de disciplina (o interdisciplina) con unos criterios bien marcados (cuanto más homogéneo, más fácil de realizar). Es el caso de NINES (una asociación para estudios del siglo XIX) ${ }^{41}$ o CHARTA. ${ }^{42}$ El éxito de estas comunidades virtuales depende de protocolos «tecno-humanísticos» comunes (el uso de estándares compartidos para depositar datos), mecanismos para la comunicación erudita a través de plataformas digitales y el reconocimiento científico de la «erudición / investigación digital». Estos protocolos tecno-humanísticos pueden comprender simplemente una puesta en común final de las publicaciones digitales (en términos técnicos, una «federación de los datos»), o

40 http://www.textgrid.de/.

41 NINES (http://www.nines.org/) ha influido a otras comunidades, como http://www.18thconnect.org/ (que estudia el siglo XVIII) y http://mesamedieval.wordpress.com/ (para investigación sobre la época medieval). El proyecto Medieval Europe-Medieval Cultures and Technological Resources es una iniciativa europea parecida (http://www.cost.eu/domains actions/isch/Actions/IS1005).

42 CHARTA es «un proyecto destinado a la publicación en red de un corpus de textos y documentos antiguos de los siglos XII al XIX de España e Hispanoamérica» (http://www.charta.es/nosotros/). 
pueden representar un intercambio más profundo de los datos de la investigación, como es el caso de IDP ${ }^{43}$ (Baumann, en prensa), que representa un sistema integrado para editar y estudiar fragmentos papirológicos; los mecanismos para la comunicación pueden incluir acuerdos sobre prioridades y sistemas de «buena práctica», dudas sobre procesos de investigación (digital o no), revisión por pares, puesta en común de herramientas digitales, y en los casos más ambiciosos, rastros de decisiones editoriales en línea; el reconocimiento científico se basa en sistemas formales (e informales) de valoración de los resultados de la investigación digital (que, al depender de una comunidad formalmente construida para promover investigación digital, es más fácil de conseguir) y la presión de las comunidades de práctica sobre las instituciones tradicionales para que reconozcan la investigación hecha con instrumentos del siglo XXI (se nota cierta falta de equilibrio entre las distintas realidades nacionales a este respecto). Para concluir, estas comunidades logran sus objetivos con mayor facilidad donde la integración digital es una oportunidad y no una obligación: varios ejemplos del pasado han demostrado que la flexibilidad y el poder trabajar en común respetando la autonomía entre investigadores y proyectos son imprescindibles.

\section{RETOS PARA LA EDICIÓN EN LA ERA DIGITAL}

Hace unos años, amplios sectores de las humanidades hubieran preguntado qué tiene que ver con temas de edición académica este estado de la cuestión en sistemas de edición digital, pero hoy en día es casi imposible pasar por alto la necesidad de una reconceptualización a escala mayor del proceso de la edición académica que salvaguarde los cimientos de la perspectiva crítica utilizando métodos y herramientas corrientes. El concepto de edición como una actividad individual y autónoma, aislada de otros procesos de la investigación, se ve cada vez más reemplazado por otro paradigma más colaborativo donde las líneas de división entre preparación de una edición, su publicación, su difusión y su lectura empiezan a borrarse o verse sustituidas por nuevas divisiones y parcelación de tareas y actividades. El gran reto para los editores del siglo xxi es decidir los límites de esta nueva

43 IDP, financiado por el Andrew W. Mellon Foundation, creó el Papyrological Navigator (http:// papyri.info/) para editar y diseminar investigación papirológica. 
visión de la edición: ¿hasta qué punto es necesario revisar el proceso en su conjunto? Las opciones a nuestro alcance dependerán mucho de cómo queramos reaccionar ante la cultura digital: dicho de otro modo, si queremos simplemente afinar los métodos que ya usamos o si vemos interesante -o incluso necesarioreplantear nuestra práctica ante un paradigma bien distinto al de la era del papel y la imprenta.

El modelo impreso, todavía muy influyente incluso en el formato digital, consigue un resultado estable (una representación «fija» de las decisiones editoriales tomadas) y a primera vista supone una vía «fácil» al seguir un camino muy bien conocido, pero tiene desventajas importantes: algunas de sus convenciones (fijadas por los límites económicos y culturales del papel impreso) pueden ser anacrónicas en el medio digital, su debilidad semántica lo pone en desventaja para usos posteriores y carece de los instrumentos para una conexión más integral con otros procesos de la investigación. La edición con modelo impreso se focaliza sobre un producto concreto, una publicación «final», pero la investigación hecha para prepararla se pierde o queda oculta detrás de ese resultado.

Si editar en la era impresa nos anima a pensar en un producto final y una publicación concreta, editar en la era digital nos invita a pensar en el proceso de la investigación de manera integral. Es el ejemplo de proyectos como REED (Records of Early English Drama) ${ }^{44}$ donde se concibe el proyecto no solamente como un lugar de producción de unos volúmenes impresos sobre la documentación del teatro clásico inglés, sino de una comunidad de investigadores marcada por su espíritu de colaboración. ${ }^{45}$ Esta visión más global ofrece una serie de ventajas teóricas —accesibilidad, diseminación, conectividad, referencialidad dinámica, uso repetido o

44 «Founded in 1975, Records of Early English Drama (REED) is an international scholarly project that is establishing for the first time the broad context from which the great drama of Shakespeare and his contemporaries grew. REED has for the last 35 years worked to locate, transcribe, and edit historical surviving documentary evidence of drama, secular music, and other communal entertainment and ceremony from the Middle Ages until 1642, when the Puritans closed the London theatres» (http://www.reed.utoronto.ca/).

45 Esta actitud transforma el concepto de lo que cuenta como resultado de la investigación: «This world community of users of REED's volumes not only thinks of REED as a place where these volumes are produced, but a place where the scholarship that the current print volumes represent is actually carried out and most clearly revealed. This centre for REED as a place of collaborative and collegial scholarship is REED's real place in the world. [...] the printed volumes are the «research output» - not the research itself» (Bradley, en prensa). 
reconstrucción de la interpretación, apertura hacia la agencia del «usuario», nuevas visualizaciones, y una visión dinámica del texto dentro de la investigación-, aunque muchas de ellas no lleguen a una realización total; pero, por otro lado, su mayor ambición requiere mayores recursos (y nuevos conocimientos «tecno-humanísticos») para realizarse, y en consecuencia no se ha llegado ni aproximado siquiera a resolver cuestiones de sostenibilidad conocidas y falta todavía una infraestructura estable a largo plazo para albergarlo. Debido en parte a la constante innovación, con fuerte influencia de intereses comerciales que favorecen una dinámica de «usar y tirar», la edad digital no ha cumplido con su promesa de salvaguardar la preservación, más bien lo contrario, en muchas áreas de la investigación. Y cualquier estrategia digital tendrá que aprovechar el dinamismo del texto digital en la preparación y la gestión de una edición, pero a la vez conseguir salidas y productos estables para la lectura y la valoración científica.

En su presentación en el seminario PROLOPE sobre la «edición crítica digital» realizada en la Universidad Autónoma de Barcelona el 18 de enero de 2013, Francesca Tomasi hizo una división entre principios, métodos y herramientas para la edición digital que quiero ampliar aquí. Podemos, bajo mi punto de vista, distinguir cinco perspectivas o aspectos de la edición digital: (1) conceptos (objetivos, público, propósito de una edición); (2) metodologías (métodos de edición y su realización en soporte digital, herramientas, flujo de trabajo, formatos de edición y su capacidad reproductora, lenguajes y estándares usados); (3) funciones y modos de acceso a la investigación (cómo se presenta la información y cómo el lector / usuario $^{46}$ interactúa con ella); (4) sus salidas en forma de publicación (formatos de publicación, grado de apertura de los métodos usados y datos creados, principios de interfaz entre investigadores y luego entre investigador y lector / usuario), y (5) infraestructuras (soportes de entrega de la información, formas de preservación). Abarcar estas perspectivas con una mínima seriedad implica, en mi opinión, compaginar al menos cuatro áreas teóricas y prácticas que tienen dinámicas distintas: la crítica textual (en sentido amplio), las teorías de la representación digital de los textos, las tendencias en la publicación moderna y los estudios sobre los procesos de la investigación erudita en la era digital.

46 Prefiero mantener aquí la ambigüedad (y la tensión constructiva) entre conceptos de «lector» y «usuario», que imperfectamente representan las dos etapas distintas en el acceso a la información exploradas en este artículo. 


\subsection{RADIOGRAFÍA DE UNA EDICIÓN DIGITAL}

Aunque hay una tradición importante de crítica textual en el medio digital (Schreibman 2013), se puede decir que hay cierta distancia entre teoría y práctica en general, y es muy poco habitual ver el desarrollo de una herramienta digital de edición a través de un proceso de debate extendido (por no hablar de abierto) sobre las necesidades teóricas y prácticas de la edición. Hay poco reconocimiento del hecho de que las tendencias tecnológicas del momento (por ejemplo, de la adopción del TEI en algunos círculos) tienden a condicionar de alguna manera el curso tomado, ${ }^{47}$ y mientras que a las humanidades les faltan herramientas estables y una buena comprensión de su uso, a las herramientas digitales les falta una base teórica que las sustente en muchos casos. No ayuda el hecho de que, aunque hay cierta presión por recalibrar nuestra actitud ante la cultura digital, no existe un claro consenso entre los profesionales de cómo la cultura digital puede cambiar la edición académica en su metodología y en sus teorías de sustento, y la naturaleza tan heterogénea de las humanidades dificulta modelos generalizables para la representación de la interpretación textual, y su plasmación en publicaciones formales.

Lejos de haber llegado a un punto estable, y sin querer menospreciar los muchos estudios y experimentos ambiciosos e ingeniosos hechos en los últimos años, la investigación en crítica textual con medios digitales apenas ha comenzado a tener eco en las humanidades, y a afrontar los desafíos humanísticos pendientes. Para ello, es preciso un mayor acercamiento de las humanidades a las teorías y prácticas de la cultura digital por un lado; y por otro, un enfoque menos anclado en cuestiones tecnológicas pragmáticas por parte de los investigadores en humanidades digitales. Es decir, hacen falta metodologías y herramientas para la edición digital con mayor integración de las teorías textuales y digitales.

Hemos visto que el término «edición digital» puede significar cualquier cosa entre una versión básica y sucedánea de un texto y una edición dinámica —creada, gestionada y publicada para una visualización múltiple con opciones sofisticadas de búsqueda y filtro de resultados-. Antes de empezar a construir una edición digital, que al menos en el plano teórico se presta al concepto de una obra dinámica que no se termina nunca (aunque puede ir mudando su piel, dejando constancia de sus distintas fases en forma de publicación fija), es muy importante aclarar sus objetivos y su perfil,

47 «Tools always shape the hand that wields them» (Spergberg-McQueen 1991:1). 
una laguna frecuente en las ediciones digitales creadas hasta ahora. En su fase inicial los aficionados a la edición digital académica a menudo han demostrado tendencia hacia las grandes visiones - evocando sistemas «comprensivos» para etiquetar todas las obras en ciencias humanas, ontologías universales, marcos integrados para la investigación - pero estas visiones, igual que algunas visiones universalistas del paradigma impreso, suelen naufragar ante la diversidad de culturas de investigación.

Quizá es pronto para llegar a una tipología estable de las ediciones digitales, aunque Siemens y otros esbozan una historia esquemática (Siemens et al. 2012a y 2012b). En todo caso la mayor capacidad reproductiva del texto digital significa que un texto puede cambiar de función operando bajo modelos distintos de edición en distintos momentos, de ahí la tendencia a considerar el texto como un archivo (que se puede complementar además por imágenes u otros objetos multimedia). Algunos proyectos, como la Edición Variorum Electrónica del Quijote (EVE-DQ) del Proyecto Cervantes de la Universidad de Texas A\&M, proponen este modelo como una «renovación de la ecdótica tradicional», más de acuerdo con el momento histórico que vivimos: "La EVE-DQ actualiza la edición de textos y la crítica textual en el proceso de incorporar un "libro" en una biblioteca de mayores dimensiones, mientras que también supone la construcción y habilitación de un archivo hipertextual» (Urbina 2005:223). Esto tiende a cambiar el papel de una edición, desde un objeto completo en su representación y presentación a un marco cargado de potencialidad pero sin ver su realización final, donde el editor tendrá que negociar cierto equilibrio entre los resultados deseados en el momento de editar y otras salidas posibles o imaginables (como el análisis geoespacial de topónimos o la agregación de antropónimos o conceptos para la búsqueda a gran escala).

Aun aceptando que cualquier edición tiene que eliminar material no deseado para llegar a una óptima organización y presentación de la investigación, ${ }^{48}$ la mayor expresividad semántica de algunos formatos digitales como TEI entra en conflicto con realidades pragmáticas de tiempo y economía. ¿Hasta qué grado debemos marcar, dar estructura, a un texto para que sirva a la investigación de hoy y mañana? A la vez, la separación entre representación semántica y presentación de un texto, posible gracias a tecnologías como XML, nos plantea una duda nueva sobre el estado ontológico de un texto y una división conceptual entre "texto como datos»y «texto como publicación», un hecho poco reconocido en la comunidad

\footnotetext{
48 «Editing makes works [in broadest sense] ... publishable... by eliminating unwanted material and organizing what remains for optimal and intelligible presentation to audiences» (Nell Smith 2009:309).
} 
de usuarios del TEI, por ejemplo, donde se plantea un cuasi-estándar que a la vez funciona como modelo de preparación, de presentación, de transferencia (a otros entornos) y de archivo. A esta relación fluida y compleja de (pre- / post-) textos en nuestra edición digital, que representa etapas claves en la vida de la investigación, cada una digna de su reconocimiento, hay que añadir el conjunto de programas — de análisis, búsqueda, filtración de resultados, transformación y presentación - que completan una edición digital, porque una edición digital es mucho más que los textos marcados, que por sí solos quedan inertes, inmóviles. Una edición digital dinámica implica una unificación de signos representativos, funciones y visualizaciones, que además depende de una infraestructura digital concreta sin garantías de preservación.

\subsubsection{ACCESO}

La era digital no solamente transforma la naturaleza de la edición, sino que también complica su creación y recepción. Los conceptos se confunden: se puede digitalizar una edición creada con criterios impresos para luego aprovecharla con todas las innovaciones tecnológicas, e igualmente se puede hacer el viaje contrario, el de editar un texto con un marco digital para sacar en versión impresa. Al contrario de los pronósticos de una inmersión total de la investigación científica en el medio digital, habitamos una realidad dual, navegando constantemente entre mundos digitales y no digitales, creando y consumiendo artefactos físicos y virtuales. El carácter flexible, huidizo, del texto digital, contrasta con la materialidad del libro, pero los dos medios tienen sus normas de diseño y de recepción determinadas por su naturaleza (física / virtual; fija / fluida; compacta / extensa) y por su estatus en la memoria cultural del ser humano, que además, al ser dialéctica, va cambiando de lugar paulatinamente. El hecho de que las metáforas digitales tomen frecuentemente conceptos de la época impresa ("escritorio», «archivo») introduce no pocos equívocos en nuestro léxico conceptual, pero se puede reconocer ya en la cultura digital «un paradigma convergente donde se unen la tecnología y la cultura», como señala Uzelac [2010].

Si bien se suele afirmar que la diseminación digital promueve el acceso digital, esta creencia no se corresponde con la realidad, al menos no hasta ahora. Si en el mundo físico el acceso del investigador está mediado por la afiliación institucional, 
las distancias geográficas a archivos y bibliotecas y los medios económicos para llevar a cabo un estudio, los contenidos digitales están mediados por un acceso al aparato digital y a una conexión a internet muy desigual, y por la gran variedad en modos de acceso (dispositivo, pantalla, navegador, app). Estos factores aseguran una experiencia muy variada entre lectores / usuarios distintos que contradice la idea de un acceso más o menos homogéneo y predecible.

\subsubsection{Modelos DEL TEXTO, REPRESENTACIÓN DIGITAL E INTERPRETACIÓN}

Al margen de si se trata de fijar una interpretación concreta para una publicación determinada o de contribuir a un archivo de textos más abiertos a un procesamiento humano o computacional posterior, está claro que se trata siempre de interpretación, para nada reducible a una objetividad esquiva. ${ }^{49} \mathrm{Si}$ en la tradición de crítica textual todavía pervive el mito del texto plano, texto limpio, que presuntamente refleja las intenciones puras del autor - como decía McGann, no existe el texto "plano», sin marcas—, ${ }^{50}$ entre los profesionales de la edición digital abunda el mito del texto "transparente», de sistemas de marcación que se abren automáticamente a reutilización por otros investigadores sin barrera, ignorando los preceptos culturales incrustados en el aparato tecnológico, y la finalidad implícita (aun si es interrogable y extensible) en cada modelo digital.

Si reconocemos que «cada codificación / representación genera, permite y regula un determinado uso y acceso al conocimiento», como aseveran Fiormonte y Schmidt (Fiormonte 2012), y que un modelo nunca es, ni puede ser, una fijación permanente del saber sino un artificio para explorar posibles interpretaciones (McCarty 2008), hay que ser conscientes de la tensión entre factores «estáticos» (los intentos de salvaguardar la investigación digital en formatos estandarizados, o al menos traducibles / recuperables) y «dinámicos» (la subjetividad de cada interpretación, además de la innovación y los fundamentos experimentales de cada modelo). A esto hay que añadir la necesidad de reconciliar la naturaleza dinámica del proceso de edición digital académica con las presiones institucionales de conseguir resultados concretos, es decir publicaciones fijas. Y la mayor variedad de marcos, formatos y

49 «Representations are inevitably partial, never disinterested [...] The representation of a text within a computer inevitably expresses an opinion about what is important in that text» (Sperberg-McQueen 1991:1).

50 «All text is marked text» (McGann 2009:198). 
herramientas hace cada vez más difícil distinguir entre elementos de investigación e instrumentos para la lectura.

\subsubsection{EnVOLTURA, TRANSMISIÓN}

Si buena parte del proceso de edición tiene lugar con instrumentos y modelos digitales, ¿cómo vamos a poder observar, captar y analizar los rastros de esta transmisión en el futuro? Es obvio que harán falta nuevos procesos de formación y herramientas para seguir la historia de una transmisión predominantemente digital y para examinar sus testimonios físicos y virtuales: una aplicación de la ciencia forense digital para las humanidades. Si no queremos ser testigos de visiones apocalípticas de la desaparición general de los fragmentos culturales del pasado, y dada la certeza casi absoluta de que muchas ediciones digitales no serán accesibles en las mismas condiciones en las que fueron creadas, tendremos que afrontar el reto de cómo reconstruir los fragmentos digitales en situaciones donde los servidores de un proyecto se han apagado por falta de financiación, donde los navegadores y dispositivos nuevos distorsionan o incluso quiebran ediciones creadas con especificaciones de otra época, o donde las herramientas dejan de recibir apoyo técnico. Y sobre todo, es menester renovar los fundamentos teóricos de la crítica textual para describir el variado surtido de envases físicos y digitales que suponen esta nueva realidad.

\subsubsection{Autoría}

En paralelo a los cambios en la concepción de la edición, el papel del editor y de la crítica textual se produce un cuestionamiento, si no recalibración, de su «autoridad» y de los signos reconocibles de una edición impresa. Las expectativas de un lector / usuario de una edición digital cambian con rapidez, y esto no solamente supone un desafío para las ediciones tradicionales, sino que también plantea un reto difícil para las ediciones digitales: ser innovadores pero con ojos dirigidos hacia la sostenibilidad; responder a una comunidad de lectores / usuarios de manera dinámica bajo limitaciones económicas importantes, y para terminar, responder al instinto participativo promovido por los medios sociales y la funcionalidad Web 2.0 (que dio protagonismo al «usuario» en la creación de contenidos 
web), sin sacrificar el criterio científico de rigor y la perspectiva crítica. ${ }^{51}$ Para complicar aún más nuestra labor, los instrumentos de evaluación no reconocen hoy en día la realidad del papel de editor, que está cada vez más distribuido, basado en la colaboración, y que a veces mezcla facetas de "experto / aficionado» y «público / privado».

\subsubsection{Evaluación DE UNA EDICIÓN DIGITAL}

La edición académica en la era digital no solamente trae consigo nuevos contenidos, sino también inéditas formas de organizar, clasificar e interactuar con la información o los datos provistos por el editor, en palabras de Presner. ${ }^{52}$ Existen importantes retos, tanto a nivel teórico (cómo repensar la labor científica en una obra altamente colaborativa que diluya o traspase las fronteras disciplinares tradicionales) como a nivel práctico (cómo identificar / elegir / formar a evaluadores capaces de examinar producción científica de una heterogeneidad ontológica enorme), y que pueden usar una amplia gama de tecnologías. Pero quizás el mayor reto es en el ahora muy cuestionado papel de la evaluación científica tradicional, y en particular el modelo de revisión anónima por pares. Es un tema demasiado largo para este artículo, que pasa por resolver las contradicciones (a menudo extremadas por los dos «bandos») entre la revisión académica por expertos y la participación activa del usuario prevalente en el modelo web, ${ }^{53}$ y que cada vez se ve más relacionado con debates generales sobre el futuro de la labor científica, que además tienen sus variaciones locales. Pero en el fondo hay planteamientos importantes sobre cómo llegar a una cultura más justa de citación en el mundo académico, ${ }^{54}$ que intenta romper barreras vistas como anacrónicas que impiden el reconocimiento justo de las aportaciones «no tradicionales» en el proceso científico,

51 Nell Smith [2009:308] propone una acomodación de los dos conceptos para beneficio del mundo científico: "If an editor chooses, the much theorized common reader can now provide immediate and actual (in contrast to imagined) critical feedback that might in turn be usefully incorporated into the work of a scholarly edition».

52 Presner [2012]: «New knowledge is not just new content but also new ways of organizing, classifying, and interacting with content». Veáse también Nowviskie [2012].

53 http://www.adelinekoh.org/blog/2013/08/29/journalofdigitalhumanitie/.

54 Tema explorado por FairCite (http://faircite.wordpress.com/) o la carta de derechos del investigador colaborador (Collaborator's Bill of Rights) (http://mediacommons.futureofthebook.org/ mcpress/offthetracks/part-one-models-for-collaboration-career-paths-acquiring-institutional-support-and-transformation-in-the-field/a-collaboration/collaborators $\%$ E2\%80\%99-bill-of-rights/). 
bien vengan de investigadores en una fase temprana de su carrera, "técnicos» u otros investigadores «extraacadémicos». Y estos debates van de la mano de una discusión sobre cómo utilizar la tecnología en la investigación científica, porque no se van a poder crear modelos estables para la edición digital sin una infraestructura académica que los nutra, empezando por el reconocimiento justo para todos los implicados.

Ha habido varios intentos de proponer criterios para evaluar las ediciones digitales - un modelo bastante exhaustivo propuesto por Patrick Sahle pasa por definir el alcance o ámbito de la edición, los marcos técnicos que la albergan, la accesibilidad, los contenidos, los objetivos y la metodología usada para llevarlos a cabo, así como la implementación de este conjunto de intenciones, y su presentación final-, pero por ahora estamos lejos de un consenso general, salvo en determinadas comunidades científicas muy avanzadas en el tema.

El último punto en este apartado, y quizás el más técnico, es cómo los marcos digitales pueden (o incluso si deben) gestionar la revisión por pares. Asociaciones como NINES proponen una evaluación que contempla tanto el aspecto digital como el aspecto humanístico, mientras que proyectos como IDP (Integrating Digital Papyrology) ofrecen una metodología innovadora que permite observar el rastro de decisiones científicas sobre una determinada edición, que además permite aportaciones «no expertas» antes de ser «aprobado» por su comité científico.

Estamos todavía muy lejos de conseguir un sistema estable para evaluar las ediciones digitales, y esto se complica más si contemplamos la posibilidad (algunos dicen "probabilidad») de que en el futuro podamos ver ediciones (o fragmentos de las mismas) que sean reutilizadas, distribuidas o agregadas en nuevos marcos creados y manejados por otros. En una visión cada vez más compartida de la investigación, ¿cómo podemos conseguir que el reconocimiento académico apropiado se traslade con cada objeto científico creado con medios digitales?

\subsection{DE LA PREPARACIÓN A LA PUBLICACIÓN}

De igual manera que se cuestiona en estos momentos el papel del investigador que prepara una edición, el papel del editor, y de las casas editoriales que publican las obras científicas, suele ponerse en tela de juicio. ${ }^{55}$ ¿Quién publica? ¿En qué formas,

55 La ambigüedad en castellano de la palabra «editar», reto lingüístico para anglófonos como yo 
en qué formatos, para qué aparatos / medios, y bajo qué modelo económico y científico? Las presiones económicas han acelerado algunas transformaciones (la migración hacia revistas digitales, por ejemplo), pero en muchos aspectos el modelo de publicación en humanidades sufre una presión fuerte en estos momentos.

Hasta ahora las ediciones digitales, y los marcos digitales que las sostienen, han seguido muy de cerca el patrón impreso, pero algunos proponen que los marcos digitales tienen que ofrecer más que un salto limitado a la eficacia. La publicación digital representa un terreno en disputa, donde varias iniciativas y procesos luchan por la hegemonía o «cuota de mercado». El resultado es una transición hacia el modelo digital por un camino inestable, que ofrece muchas opciones -interactividad, acceso a datos primarios de la investigación, acceso a información sobre usuarios, relaciones con una comunidad de lectores, publicación parcial y por fases, conexión con objetos multimedia, agregación de contenidos- pero que no cristaliza en una oferta concreta, fácilmente aprovechable por la comunidad científica.

En su análisis del artículo científico, Breure, Voorbij y Hoogerwerf proponen un nuevo modelo de publicación, que voy a traducir como "publicación aumentada" (Breure et al. 2011:3), un concepto que cubre una amplia gama de posibilidades, desde una publicación tradicional con algún elemento interactivo conectado de una forma limitada, hasta publicaciones que contienen una estructura multimedia y no-linear menos anclada en la tradición textual (proponen el denominador Rich Internet Publications, para reemplazar al concepto de enhanced publications). Queda mucho camino todavía, pero ya vemos indicios de que algunos investigadores se van liberando de las premisas impresas, pues la era digital nos permite casi «empezar desde cero» en cuanto a las posibles visualizaciones de la investigación.

Esto favorece especialmente a áreas de la ciencia históricamente mal servidas por un tratamiento textual primario, como el estudio del teatro: «los medios electrónicos ofrecen un sitio / escenario para las actuaciones», nos sugiere Worthen [2003:173], y aunque los primeros años de edición digital generalmente no vieron realizarse este loable sueño, son cada vez más frecuentes las ediciones que se alejan de esta tradición, sin llegar todavía al juego entre textualidad, visualidad y

que quieran contrastar lo que en inglés serían «editing» y «publishing», es testamento de la imprudencia de hacer una separación estricta entre la preparación de una edición y su presentación en una publicación formal. 
actuación que propone Worthen. ${ }^{56}$ Quizás, además, la relación problemática para los estudios del teatro entre el texto de la obra teatral y sus múltiples actuaciones nos sirve de paradigma para situar algunos modelos de la edición digital en el plano teórico: el modelo base en TEI / XML (equivalente al texto dramático) y sus múltiples visualizaciones (léase representaciones). Al problema del «texto» y su «actuación» se añade el reto de la traducción: en el teatro, la traducción entre idiomas, periodos o contextos sociales; y en la edición digital, entre programas informáticos, estándares técnicos y universos científicos o culturales de diversa tradición epistemológica.

Hay una clara contradicción entre la necesidad de ofrecer interfaces sencillas y el deseo de crear publicaciones que aprovechen las posibilidades del medio digital -la contextualización dinámica, ediciones extratextuales, las búsquedas dinámicas, la autopublicación, la publicación como foco de discusión en línea, las ediciones que se aprovechan del análisis geoespacial— donde la «unidad de significación» no necesariamente será un «libro», sino que puede ser un fragmento de interpretación científica dentro de archivos o colecciones dinámicos.

A pesar de que algunos de los aparatos y formatos digitales introducidos en los últimos años, casi todos por potencias tecnológicas como Amazon o Apple, han tenido un efecto notable sobre el consumo de la letra escrita en general, su adopción para la investigación, más allá de tareas básicas como la simple lectura, ha sido más modesta. Faltan estudios para entender mejor si los hábitos de lectura de la comunidad científica en humanidades experimentan cambios profundos a causa de la tecnología digital, ${ }^{57}$ pero parece probable que estos seguirán las tendencias generales de la comunidad científica, que paulatinamente se adapta a los paradigmas nuevos. Por ahora, las estrategias de publicación académica han ignorado las tabletas y dispositivos móviles por lo general, pero eso puede cambiar rápido en los próximos años, y esto obligará a replantear los pilares fundacionales de las publicaciones. En este panorama de múltiples visualizaciones del mismo texto, en múltiples formatos, y en múltiples medios, ¿cuál es la base para una evaluación científica sistemática?

56 Worthen [2003:197]: «The digital metamorphoses of the screen ought to stage an awareness of the interplay between textualities, visualities, and performances».

57 http://www.inke.ca/. 


\subsection{LA EDICIÓN DENTRO DEL PROCESO DE LA INVESTIGACIÓN}

Este artículo ha señalado una brecha importante entre edición digital como estrategia de simple difusión y edición digital como parte de un modelo más amplio, donde ya no nos tenemos que limitar a comunicar los resultados del proceso de nuestra investigación, sino que podemos (1) ofrecer nuestros materiales y datos para el uso por otros (bajo sistemas que vigilen los derechos de autor); (2) crear nodos de conexión actualizables entre nuestro trabajo y la investigación de otros; y (3) hacer enlaces dinámicos a taxonomías u otros sistemas de referencia en línea, facilitando comentarios y anotaciones dinámicas de un público más amplio que nuestra comunidad científica más cercana, y permitiendo a los lectores/usuarios «ser testigos de las etapas intermedias del proceso de descubrimiento científico». ${ }^{58}$ Es un modelo que vislumbra una nueva práctica de la investigación que ya se empieza a ver en algunas áreas científicas, y que comprende acceso abierto al cultivo y a los frutos de la investigación (no solamente las publicaciones producidas por ella), colaboración e interacción, apropiación de algunas de las características positivas de los medios sociales (siempre manteniendo el rigor académico), y nuevas maneras de buscar, seguir y sopesar la información a través de entornos virtuales conectables entre sí.

En este sentido, el libro blanco para una edición digital de los archivos del teatro Fortune (Boyd y Bradley 2012) hace una evaluación global de los protocolos, prácticas editoriales, flujos de trabajo, formatos y software necesarios para crear un prototipo de edición digital académica dentro de un concepto global de la investigación. Tomando como material de prueba los registros dramáticos de Jessica Freeman relacionados con el teatro Fortune de Londres, lugar simbólico del teatro clásico inglés — que serán publicados en una colección futura del proyecto REEDel estudio ha propuesto un modelo de edición que integra metodología tradicional con marcación estructurada en TEI, gestión y control de las versiones de cada texto, manejo sistemático de códigos históricos (at-codes) del proyecto REED para registrar cabeceras y notas, trabajo por fases editoriales, salidas múltiples (plena transcripción diplomática, transcripción crítica del editor y generación automática de varias notas), la creación inicial de un sistema para gestionar las entidades (personas, lugares y temas) mencionados en los escritos, y que pueden servir para generar índices dinámicos y campos de búsqueda, y la posible creación de glosarios

58 Breure et al. [2011]: «Be witness to intermediary stages of scientific discover process». 
y bibliografías dinámicos en el futuro. Propone todo esto dentro de un paradigma dual (digital / impreso) de la publicación que, usando la misma fuente de material editado en TEI / XML, tiene varias salidas digitales (que pueden variar tanto en forma científica como en medio tecnológico) y que puede a la vez crear los volúmenes tradicionales de REED en formato impreso. Este modelo incluso se presta a publicaciones más centradas en el «lector / usuario», que le permiten crear sus propias colecciones de materiales, que en palabras del informe funcionan como una "cesta", desde la cual se puede añadir y agregar varios contenidos para crear publicaciones impresas (Boyd y Bradley 2012:34).

Como hemos visto, el panorama de la edición digital presenta múltiples modelos editoriales y técnicos que suponen una oferta a veces confusa, complicado por los cambios rápidos en la tecnología que provocan tensiones entre el deseo de innovar y la necesidad de crear trabajo científico fijo, estable y citable. Aunque una migración a la edición digital de una forma u otra parece inevitable, hay serias cuestiones de sostenibilidad lejos de estar resueltas. El trabajo en grupos mayores, a veces formalizados en asociaciones profesionales como NINES y CHARTA puede ser una vía, pero tal vez la clave esté en promover un diálogo más cercano entre los actores más comprometidos con la creación, publicación y preservación de la ciencia humana - es decir, investigadores académicos, archivos, bibliotecas académicas y editoriales académicas- Hay que recordar que cada actor / sector tiene su propia experiencia en crear infraestructura digital, pero rara vez se comunican lo suficiente para conseguir un alineamiento de prácticas.

\section{Hacia nuevos modelos de Edición EN LA ERA Digital}

Este estudio ha pretendido esbozar algunos de los retos que nos esperan todavía para encontrar un espacio estable para la edición académica en la edad digital. Como es natural en un momento histórico de esta naturaleza, que a pesar del carácter rápido y vertiginoso de sus cambios representa una larga transición desde la edad de la palabra impresa, podemos tener la percepción de que el debate sobre el futuro de la edición científica suscita más preguntas que respuestas, pero insisto en que nos urge como humanistas hacer frente a estas preguntas, para no ser marginados por una historia que está en estos momentos muy lejos de nuestro control. 
Es habitual ahora en la ciencia de la información hablar de "ecosistemas digitales», y hay algunos, como Jason Kelly, que proponen nuevas «ecologías» para la investigación en la era digital, argumentando que situarnos en este entorno más extenso nos puede ayudar a entender qué será de nuestras disciplinas en el futuro. ${ }^{59} \mathrm{He}$ querido sugerir que faltaría un replanteamiento largo y profundo de la manera en que creamos y producimos ediciones académicas en la era digital, y que esto requiere nuevos modelos de colaboración entre investigadores en humanidades, ciencias de la información y humanidades digitales. Debe ser, a mi juicio, una colaboración donde se dé reconocimiento adecuado a la investigación técnica pero a la vez donde los criterios humanísticos tengan un papel determinante también en el desarrollo de nuevos caminos hacia la construcción del saber.

Hasta ahora, y aunque algunas empresas tecnológicas como Apple hacen alarde de sus raíces humanísticas (véase Lehrer 2011), las herramientas e infraestructuras que manejamos nos llegan de prestado de otros mundos científicos y profesionales, con todos los problemas de transposición de modelos y metodologías que conocemos. Será difícil avanzar mientras amplios sectores en las humanidades vean a «lo técnico» solamente como los medios para un fin (un fin además, en este momento, ampliamente limitado), y solamente vamos a conseguir una mejor convergencia de las humanidades y la tecnología si agilizamos mejor la comunicación entre los dos mundos científicos.

Es evidente que un investigador no puede ser experto en todo, y que hay que evitar la dilución de las especialidades, uno de los mayores riesgos de este florecimiento de nuevas destrezas y habilidades científicas propuestas, y exigidas, por la edad digital. Pero muchos investigadores del campo de las humanidades digitales opinan que una separación absoluta entre filólogos (investigador, dirigente científico) y técnicos (apoyo humano al servicio de las directrices del humanista) empobrece los resultados de la colaboración, que se fortalece cuando la información y la valoración científica fluye en las dos direcciones (Bradley 2009:16). Aunque encontrar soluciones técnicas estables es, evidentemente, un requisito importante para dotar de instrumentos a los objetivos humanísticos en la era digital, el ciclo de innovación constante requiere «una evolución de herramientas e interfaces... que es, en igual

59 Kelly [2012]: «Ecologies show connections, interactions, feedbacks, and emerging behaviors between seemingly disparate agents and systems. Understanding where we are positioned in a larger environment will assist us in working out what our discipline might become over the next century». 
medida, interfaz hacia una publicación académica y una expresión de su compromiso intelectual y científico con el texto». ${ }^{60}$ Este compromiso requiere mirar críticamente hacia el pasado (papel histórico de las humanidades) y mirar críticamente hacia el futuro (papel histórico de las humanidades digitales), y la apertura actual hacia las humanidades digitales es una oportunidad histórica que ni humanistas ni humanistas digitales deben desperdiciar. La investigación en crítica textual que no atienda a los paradigmas digitales tiene poco futuro, pero la investigación en humanidades digitales que no atienda a la historia de las ciencias humanas carece de pasado, de fundamento. ${ }^{61}$

\section{Agradecimientos}

Quiero agradecer a las siguientes personas, que han respondido a varias consultas durante mi investigación - Paul Caton, Anna Jordanous, John Lavagnino, John O’Neill, Elena Pierazzo, Roberto Rosselli di Turco y Paul Vetch-y a todos los participantes en los proyectos de DDH aquí mencionados, destacando a Eleonora Litta y Geoffroy Noel por su trabajo en Early English Laws. En especial, debo reconocer a Carmen Isasi Pérez, Antonio Rojas Castro y Nàdia Revenga García por leer las primeras versiones de este artículo; cualquier defecto del mismo es mío, pero agradezco sus muy valiosos comentarios.

60 «But remodeling requires an evolution of tools and interfaces, a perpetual cycle of invention and reinvention, which is as much an expression of intellectual and scholarly engagement with text as it is the interface to a scholarly publication», argumenta Van Zundert [2013].

61 Con perdón a Van Zundert [2013], que presenta una oposición distinta a la que propongo aquí: "And this is the paradox: textual scholarship that ignores the digital paradigm has little future, but the textual scholarship community is certainly at this point in time not computer literate enough to meet its own digital needs». 


\section{BIBLIOGRAFÍA}

BARAIBAR, Álvaro y Shai Cohen, «Nuevas tecnologías y redes sociales en la investigación en Humanidades", La Perinola, XVI (2012), pp. 155-164. Accesible en http:// dspace.unav.es/dspace/bitstream/10171/23720/1/Baraibar Cohen Perinola.pdf.

Baumann, Ryan, «The Son of Suda On-Line», Bulletin of the Institute of Classical Studies (en prensa).

Borgman, Christine, «The Digital Future is Now: A Call to Action for the Humanities», Digital Humanities Quarterly, III 4 (2009). Accesible en http://digitalhumanities.org/dhq/vol/3/4/000077/000077.html.

Boyd, Jason y John Bradley, con Carolyn Black, Sally-Beth MacLean y Paul Vetch para el equipo del proyecto Fortune Theatre Records, «The Fortune Theatre Records: A Prototype Digital Edition, Records of Early English Drama (REED): White Paper» (2012). Accesible en http://www.reed.utoronto.cal FortuneWhitePaper.pdf.

BRADLEY, John, «What is this thing called REED? Capturing and expressing REED's essence in a digital future», en Envisioning REED in the Digital Age, eds. J. Boyd y S-B. MacLean eds., New Technologies in Medieval and Renaissance Studies/Iter at the University of Toronto, Toronto (en prensa).

BradLEy, John, "What the Developer Saw: an Outsider's View of Annotation, Interpretation and Scholarship», Digital Studies / Le champ numérique, I 1 (2009). Accesible en http://www.digitalstudies.org/ojs/index.php/ digital studies/article/view/143/202.

Breure, Leen, Hans VoorbiJ y Maarten Hoogerwerf, «Rich Internet Publications: 'Show What You Tell'», Journal of Digital Information, XII 1 (2011). Accesible en http://journals.tdl.org/jodi/index.php/jodi/article/view/1606.

Cayless, Hugh, Charlotte Rouché, Tom Elliott y Gabriel Bodard, «Epigraphy in 2017», Digital Humanities Quarterly, III 1 (2009). Accesible en http://www. digitalhumanities.org/dhq/vol/3/1/000030/000030.html.

Dacos, Marin y Pierre Mounier, L'Édition électronique, Éditions la Découverte, París (2010).

Fiormonte, Domenico y Desmond SchmidT, «El problema de la representación digital de los textos: más allá del XML», presentación de Domenico Fiormonte y Demond Schmidt para el seminario «Humanidades Digitales: Edición y Difusión», 
Universidad de La Coruña, 2-3 de julio de 2012. Accesible en http://c.scholarpress.es/index/hhdd/hded/paper/view/5/4.

Fiormonte, Domenico, Valentina Martiradonna y Desmond Schmidt, «Digital Encoding as a Hermeneutic and Semiotic Act: The Case of Valerio Magrelli», Digital Humanities Quarterly, IV 1 (2010). Accesible en http://digitalhumanities.org/dhq/vol/4/1/000082/000082.html.

Fraistat, Neil y Steven E. Jones, «Editing environments: the architecture of electronic texts», Literary \& Linguistic Computing, XXIV 1 (2009), pp. 9-18.

GIBBs, Fred y Trevor Owens, «Building Better Digital Humanities Tools: Toward broader audiences and user-centered designs», Digital Humanities Quarterly, VI 2 (2012). Accesible en http://www.digitalhumanities.org/dhq/vol/6/2/000136/000136.html. HAGEL, Stefan, «The Classical Text Editor. An attempt to providing for both printed and digital editions», Digital Philology and Medieval Texts, eds. A. Ciula y F. Stella, Pacini Editore, Pisa (2007), pp. 77-84. Accesible en http://www.infotext.unisi.it/upload/DIGIMED06/book/hagel.pdf.

Harvey, P.D.A., Editing Historical Records, The British Library, Londres (2001). Hedges, Mark, Heike Neuroth, Kathleen M. Sмith, Tobias Blanke, Laurent Romary, Mark Küster y Malcolm ILlingworth, "TextGrid, TEXTvre, and DARIAH: Sustainability of Infrastructures for Textual Scholarship», Journal of the Text Encoding Initiative, V (2013). Accesible en http://jtei.revues.org/774.

KeLLy, Jason M., «An Ecology for Digital Scholarship», blog con el texto de la presentación de Jason M. Kelly en Digital History Seminar, IHR Digital (2012). Accesible en http://ihrdighist.blogs.sas.ac.uk/2012/12/10/67/.

Lavagnino, John, «The digital text and beyond», The Cambridge World Shakespeare Encyclopedia, ed. B. R. Smith, Cambridge University Press (en prensa).

LeHrer, Jonah, «Technology Alone Is Not Enough», New Yorker, 7 de octubre de 2011. Accesible en http://www.newyorker.com/online/blogs/newsdesk/2011/10/steve-jobs-pixar.html.

LiU, Alan, "From Reading to Social Computing», Literary Studies in the Digital Age (2013). Accesible en http://dlsanthology.commons.mla.org/ from-reading-to-social-computing/. $<$ http://dx.doi.org/10.1632/lsda.2013>

Mackenzie, David, A Manual of Manuscript Transcription for the Dictionary of the Old Spanish Language, Hispanic Seminar of Medieval Studies, Madison, 
1997, 5. ${ }^{a}$ edición revisada y ampliada por R. Harris-Northall. Accesible en http://www.hispanicseminary.org/manual-es.htm.

McCARty, Willard, «Knowing ... : Modeling in Literary Studies», en A Companion to Digital Literary Studies, eds. S. Schreibman y R. Siemens, Blackwell, Oxford, 2008. Accesible en http://www.digitalhumanities.org/companionDLS/.

McGann, Jerome, «Marking Texts of Many Dimensions», en A Blackwell Companion to Digital Humanities, eds. S. Schreibman, R. Siemens y J. Unsworth, Blackwell, Oxford (2004), pp. 198-217.

<http://dx.doi.org/10.1111/b.9781405103213.2004.00019.x>

$<$ http://dx.doi.org/10.1002/9780470999875>

$<\mathrm{http}$ ://dx.doi.org/10.1111/b.9781405103213.2004.x>

McIlroy, Thad, "Has XML Failed Publishing?», en blog The future of publishing, de Thad McIlroy, 2012. Accesible en http://thefutureofpublishing.com/2012/10/ xml-failed-publishing/.

Moyles, Martin, Justin TonRa y Valerie Wallace, «Manuscript Transcription by Crowdsourcing: Transcribe Bentham», Liber Quarterly, XX 3/4 (2011). Accesible en http://liber.library.uu.nl/index.php/lq/article/view/ URN\%3ANBN\%3ANL\%3AUI\%3A10-1-113603.

MuELLER, Martin, Letter about the TEI, 2011. Accesible en http://ariadne.northwestern.edu/mmueller/teiletter.pdf.

MuÑoz Pons, Carlos, «El proceso de edición digital en Artelope y CTCE», Teatro de palabras: revista sobre teatro áureo, VII (2013), pp. 483-496. Accesible en http://www.uqtr.ca/teatro/teapal/TeaPalNum07.html.

Nell Smith, Martha, «Electronic Scholarly Editing», A Blackwell Companion to Digital Humanities, eds. S. Schreibman, R. Siemens y J. Unsworth, Blackwell, Oxford (2004), pp. 306-322.

Nowviskie, Bethany, «Evaluating Collaborative Digital Scholarship (or, Where Credit is Due)», Journal of Digital Humanities, I 4 (2012). Accesible en http://journalofdigitalhumanities.org/1-4/evaluating-collaborative-digital-scholarship-by-bethany-nowviskie/.

Oтт, Wilhelm, «Modularity, Professionality, Integration: A Conception Revisited», ponencia para Congreso ALLC-ACH, Oxford (1992). Accesible en http://www. tustep.uni-tuebingen.de/tustep ox92.html.

Presner, Todd, «How to Evaluate Digital Scholarship», Journal of Digital Humanities, I 4 (2012). Accesible en http://journalofdigitalhumanities. org/1-4/how-to-evaluate-digital-scholarship-by-todd-presner/ 
PrIEM, Jason, «Scholarship: Beyond the paper», Nature, CDXCV (2013), pp. 437-440. Accesible en http://www.nature.com/nature/journal/v495/n7442/full/495437a. html?WT.ec id=NATURE-20130328.

ProLoPe, grupo de investigación, La edición del teatro de Lope de Vega: las «Partes» de comedias, Criterios de edición, Universitat Autònoma de Barcelona, Bellaterra, 2008.

RENEAR, Allen, «Text Encoding», en A Blackwell Companion to Digital Humanities, eds. S. Schreibman, R. Siemens y J. Unsworth, Blackwell, Oxford (2004), pp. 218-239. $<$ http://dx.doi.org/10.1002/9780470999875> $<$ http://dx.doi.org/10.1111/b.9781405103213.2004.00020.x> $<$ http://dx.doi.org/10.1111/b.9781405103213.2004.x>

RoBinson, Peter, «Current issues in making digital editions of medieval texts—or, do electronic scholarly editions have a future?», Digital Medievalist, I (2005). Accesible en http://www.digitalmedievalist.org/journal/1.1/robinson/.

SAHLE, Patrick, en colaboración con Georg Vogeler y los miembros de IDE, Kriterien für die Besprechung digitaler Editionen, Version 1.0 (2012). Accesible en http:// www.i-d-e.de/aktivitaeten/reviews/kriterien-version-1\#sthash.mwNcFLCv.dpuf.

SÁnchez-Prieto BorJa, Pedro, Cómo editar los textos medievales, Arco Libros, Madrid, 1998.

Schreibmann, Susan, «Digital Scholarly Editing», Literary Studies in the Digital Age (2013). Accesible en http://dlsanthology.commons.mla.org/ digital-scholarly-editing/. $<$ http://dx.doi.org/10.1632/lsda.2013>

Shildingsburg, Peter, «How Literary Works Exist: Convenient Scholarly Editions», Digital Humanities Quarterly, III 3 (2009). Accesible en http://digitalhumanities.org/dhq/vol/3/3/000054/000054.html.

Siemens, Ray, Meagan Timney, Cara Leitch, Corina Koolen y Alex Garnett, «Pertinent Discussions Toward Modeling the Social Edition: Annotated Bibliographies», Digital Humanities Quarterly, VI 1 (2012). Accesible en http://digitalhumanities.org/dhq/vol/6/1/000111/000111.html.

Siemens, Ray, Meagan Timney, Cara Leitch, Corina Koolen y Alex Garnett, con ETCL, INKE and PKP Research Groups, «Toward Modelling the social edition: An approach to understanding the electronic scholarly edition in the context of new and emerging social media», LLC. The Journal of Digital Scholarship in the Humanities, XXVII 4 (2012).

Spence, Paul, Carmen Isasi Martínez, Elena Pierazzo e Irene Vicente Miguel, «Cruzando la brecha: la marcación digital con criterios filológicos», Nuevas 
perspectivas para la edición y el estudio de documentos hispánicos antiguos, eds. P. Sánchez-Prieto Borja y M. J. Torrens Álvarez, Peter Lang, Berna, 2012. Spence, Paul, «Teatro clásico y humanidades digitales: el cruce entre método, proceso y nuevas tecnologías», Teatro de palabras: revista sobre teatro áureo, VII (2013), pp. 9-38. Accesible en http://www.uqtr.ca/teatro/teapal/TeaPalNum07.html.

Sperberg-McQueen, C. M., «Text in the Electronic Age: Textual Study and Text Encoding, with Examples from Medieval Texts», Literary and Linguistic Computing, VI 1 (1991), pp. 34-46.

<HTTP://DX.DOI.ORG/10.1093/LLC/6.1.34>

Standing Committee for the Humanities (SCH), «Research Infrastructures in the Digital Humanities», Science Policy Briefing, European Science Foundation (2011). Accesible en http://www.esf.org/fileadmin/Public documents/Publications/ spb42 RI DigitalHumanities.pdf.

Urbina, Eduardo, Erica Pasquel, Richard Futura, Carlos Monroy, Jie Deng y Neal Audenauert, «Humanidades digitales, crítica textual y la edición variorum electrónica del Quijote (EVE DQ)», Actas de la A.ISPI.I (2005), pp. 223-235. Accesible en http://cvc.cervantes.es/literatura/aispi/pdf/21/I 20.pdf.

UZELAC, Aleksandra, «La cultura digital, un paradigma convergent on s'uneixen la tecnologia i la cultura: reptes per al sector cultural», Digithum, XII (2010), pp. 25-31. Accesible en http://digithum.uoc.edu/ojs/index.php/digithum/article/view/n12-uzelac.

VAnhoutte, Edward, «So you think you can edit? The Masterchef edition», en blog The Mind Tool: Edward Vanhoutte's blog, 2011. Accesible en http://edwardvanhoutte.blogspot.co.uk/2011/10/so-you-think-you-can-edit-masterchef.html.

VAN ZUNDERT, Joris, «Careful what you wish for», en blog Braenden's Flow de Joris van Zundert, 2013. Accesible en http://brandaen. huygensinstituut.nl/?p=497.

VeTch, Paul, «From Edition to Experience: Feeling the Way towards User Focussed Interfaces», en Electronic Publishing: Politics and Pragmatics, ed. G. Egan, Medieval and Renaissance Texts and Studies / ITER, Toronto (2010), pp. 163-176. Vieillard, Françoise y Olivier Guyotjeannin eds., Conseils pour l'édition des textes médiévaux, I-III, Comité des Travaux Historiques et Scientifiques y École Nationale des Chartes, París (2001).

Worthen, W. B., Shakespeare and the Force of Modern Performance, Cambridge University Press, Cambridge (2003). $<$ http://dx.doi.org/10.1017/CBO9780511484087> 


\section{ENLACES CITADOS}

Todos los sitios webs aquí mencionados fueron consultados el 23 de septiembre de 2013.

18thConnect: http://www.18thconnect.org/.

BestPracticesforTEI in Libraries:http://www.tei-c.org/SIG/Libraries/teiinlibraries/.

CATMA (Computer Aided Textual Markup \& Analysis): http://www.catma.de/.

CLARIN: http://www.clarin.eu/.

CTE (Classical Text Editor): http://cte.oeaw.ac.at/.

Collaborators' Bill of Rights: http://mediacommons.futureofthebook.org/mcpress/ offthetracks/part-one-models-for-collaboration-career-paths-acquiring-institutional-support-and-transformation-in-the-field/a-collaboration/ collaborators $\% \mathrm{E} 2 \% 80 \% 99$-bill-of-rights/.

Critical Apparatus chapter in TEI P5 Guidelines: http://www.tei-c.org/release/doc/ tei-p5-doc/en/html/TC.html.

Critical Appartus Workgroup, TEI: http://wiki.tei-c.org/index.php/ Critical Apparatus Workgroup.

CWRC-Writer: An In-Browser XML Editor: http://www.cwrc.ca/projects/ infrastructure-projects/technical-projects/cwrc-writer/.

DARIAH: dariah.eu/.

Department of Digital Humanities, King's College London: http://www.kcl.ac.uk/ artshums/depts/ddh/index.aspx.

EPIDOC (Epigraphic Documents in TEI XML): http://sourceforge.net/p/epidoc/wiki/ Homel.

Edición electronica de La entretenida: http://entretenida.outofthewings.org, editada por John O'Neill.

Faircite: http://faircite.wordpress.com/.

Guide for Editors, Early English Laws: http://www.earlyenglishlaws.ac.uk/edit/ guide-editors/.

Juxta software: http://www.juxtasoftware.org/.

Kiln: http://github.com/kcl-ddh/kiln.

Medieval Electronic Scholarly Alliance: http://mesamedieval.wordpress.com/.

Medieval Europe-Medieval Cultures and Technological Resources: http://www.cost. eu/domains actions/isch/Actions/IS1005. 
Medieval Nordic Text Archive: http://www.menota.org/forside.xhtml.

NINES (Nineteenth-century Scholarship Online): http://www.nines.org/.

Perseus Digital Library: http://www.perseus.tufts.edu.

Proyecto Early English Laws: http://www.earlyenglishlaws.ac.uk/.

Proyecto Henrik Ibsens Skrifter: http://www.ibsen.uio.no.

Proyecto Early Americas Digital Archive: http://mith.umd.edu/eada/intro.php.

Proyecto Inke: http://inke.cal.

Proyecto Orlando: http://www.artsrn.ualberta.ca/orlando/.

Proyecto Out of the Wings: http://outofthewings.org/.

Proyecto Papyri.info: http://papyri.info/.

Proyecto REED (Records of Early English Drama): http://www.reed.utoronto.cal.

Proyecto TAPAS Project: http://tapasproject.org/.

Proyecto TEI by Example: http://www.teibyexample.org/.

Proyecto The Gascon Rolls (1317-1468): http://gasconrolls.org.

Proyecto Transcribe Bentham: http://blogs.ucl.ac.uk/transcribe-bentham/.

Red CHARTA (Corpus Hispánico Americano en la Red: Textos Antiguos): http://www. charta.es.

Scripto: http://scripto.org/.

TC12, TC/12, Patrimonio teatral clásico español. Textos e instrumentos de investigación: http://tc12.uv.es/.

TEI Boilerplate: $\underline{h t t p: / / d c l . s l i s . i n d i a n a . e d u / t e i b p / . ~}$

TEI Customization: http://www.tei-c.org/Guidelines/Customization/.

TextGrid: http://www.textgrid.de/.

TILE 1.0 (Text-Image Linking Environment): mith.umd.edu/tile/.

Tustep (TUebingen System of TExt Processing tools): http://www.tustep.uni-tuebingen.de/tustep eng.html.

UVic Image Markup Tool Project: http://tapor.uvic.ca/ mholmes/image markup/.

Valuing / Evaluating DH Practice, Carolina Digital Humanities Initiative: http://digitalhumanities.unc.edu/resources/valuing-evaluating-dh-practice/?doing wp cr on $=1374843194.6683659553527832031250$.

Virtual research environment programme, Jisc: http://www.jisc.ac.uk/whatwedo/ programmes/vre.aspx. 